\title{
Ultrafast and Highly Sensitive Photodetectors With Recessed Electrodes Fabricated on Low-Temperature-Grown GaAs
}

\author{
M. Mikulics, S. Wu, M. Marso, R. Adam, A. Förster, A. van der Hart, P. Kordoš, H. Lüth, and Roman Sobolewski
}

\begin{abstract}
We have fabricated and characterized ultrafast metal-semiconductor-metal (MSM) photodetectors with recessed electrodes, based on low-temperature-grown GaAs. The new recessed-electrode MSM geometry led to an improved electric-field distribution inside the photodetector structure and resulted in a $25 \%$ breakdown voltage and sensitivity increase with simultaneous four-fold reduction of capacitance, as compared to the identical MSM devices with planar electrodes. Time-resolved studies performed using $\mathbf{1 0 0 - f s - d u r a t i o n ~ l a s e r ~ p u l s e s ~ s h o w e d ~ t h a t ~}$ recessed-electrode MSMs exhibited 1.0-ps-wide photoresponse transients with no slow after-pulse tails and their photoresponse time was $0.9 \mathrm{ps}$. The improved transient photoresponse parameters are the main advantages of the recessed-electrode geometry.
\end{abstract}

Index Terms-Low-temperature-grown GaAs (LT-GaAs), metal-semiconductor-metal(MSM) diode, metal-semiconductormetal (MSM) photodetectors, recessed electrode structure, ultrafast photodetectors.

T HE low-temperature-grown GaAs (LT-GaAs) semiconductor has been well known for more than a decade for its subpicosecond carrier lifetime and acceptable mobility and it has been used very often for the fabrication of ultrafast optoelectronic photodetectors and photomixers [1], [2]. In the published data discussing metal-semiconductor-metal (MSM) photodetectors, their electrodes are typically fabricated on the surface of the LT-GaAs material [3], [4], and improvements to enhance the detector photoresponse efficiency have been mainly focused on increase of the device active area and on the optimization of the MSM interdigitated structure.

The aim of this letter is to present fabrication and properties of modified LT-GaAs MSM photodetectors. We report on the devices with recessed metal electrodes and demonstrate that this new geometry results in significant improvements of the

Manuscript received November 8, 2005; revised January 12, 2006. This work was supported in part by National Science Foundation (NSF) Grant INT-0078949 and in part by Corning Inc. Additional support was provided by the Center for Electronic Imaging Systems, a NYSTAR-designated New York Center for Advanced Technology.

M. Mikulics is with the Max-Plank-Institute for Radioastronomy, D-53121 Bonn, Germany, also with the Institute of Thin Films and Interfaces, Research Centre Jülich, D-52425 Jülich, Germany, and also with Institut fuer Hochfrequenztechnik, Technische Universitaet Braunschweig, D-38106 Braunschweig, Germany.

S. Wu is with the Department of Physics and Astronomy and the Laboratory for Laser Energetics, University of Rochester, Rochester, NY 14627-0231 USA.

M. Marso, R. Adam, A. Förster, A. van der Hart, P. Kordoš, and H. Lüth are with the Institute of Thin Films and Interfaces, Research Centre Jülich, D-52425 Jülich, Germany.

R. Sobolewski is with the Department of Electrical and Computer Engineering and the Laboratory for Laser Energetics, University of Rochester, Rochester, NY 14627-0231 USA (e-mail: roman.sobolewski@ rochester.edu).

Digital Object Identifier 10.1109/LPT.2006.871696
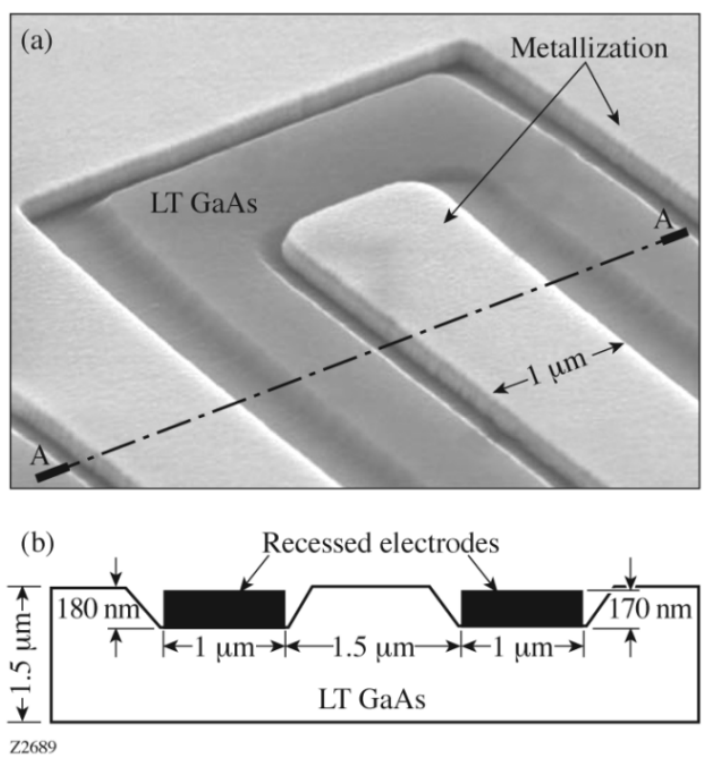

Fig. 1. (a) Scanning electron micrograph of an MSM LT-GaAs photodetector with recessed electrodes; (b) cross section A-A showing the structure dimensions (the LT-GaAs groove and the Ti-Au electrodes are not to scale).

MSM photodetector performance, as compared to conventional structures fabricated with planar electrodes. MSMs with surface electrodes typically suffer from a nonuniform distribution of the electric field in an active layer of the photodetector and a low voltage breakdown over the device surface. The main idea behind our concept is to partially bury the electrodes, so the additional active material is located in the region of the highest electric field. A similar approach has been recently implemented in the form of MSMs with trench electrodes [5], [12], [13].

Our MSM structures consist of Ti-Au electrodes with a thickness of $10 \mathrm{~nm}$ of Ti and $160 \mathrm{~nm}$ of Au, deposited on a $1.5-\mu \mathrm{m}$ -thick undoped LT-GaAs epitaxial film, grown by molecular beam epitaxy on top of a 300-nm-thick AlAs interlayer on a 2-in semi-insulating GaAs wafer. The growth process was performed at $250{ }^{\circ} \mathrm{C}$ (thermocouple reading from the substrate side), followed by in situ isothermal annealing at $600{ }^{\circ} \mathrm{C}$ for $10 \mathrm{~min}$. The devices had the dimensions 20 by $30 \mu \mathrm{m}$ and were patterned using a conventional photolithography and liftoff technique [6]. The MSM finger width of our devices was $1 \mu \mathrm{m}$ and the finger spacing was $1.5 \mu \mathrm{m}$, as presented in Fig. 1. The "V" profile groove for metal electrodes was pre-etched using an anisotropic wet etch $\mathrm{H}_{3} \mathrm{PO}_{4}: \mathrm{H}_{2} \mathrm{O}_{2}: \mathrm{H}_{2} \mathrm{O}(3: 1: 50)$ [7]. The groove depth $d$ in our tested photodetectors varied from 60 to $300 \mathrm{~nm}$ and the wet-chemical etch did not degrade the LT-GaAs material, although we expect some near-surface damage of the groove 


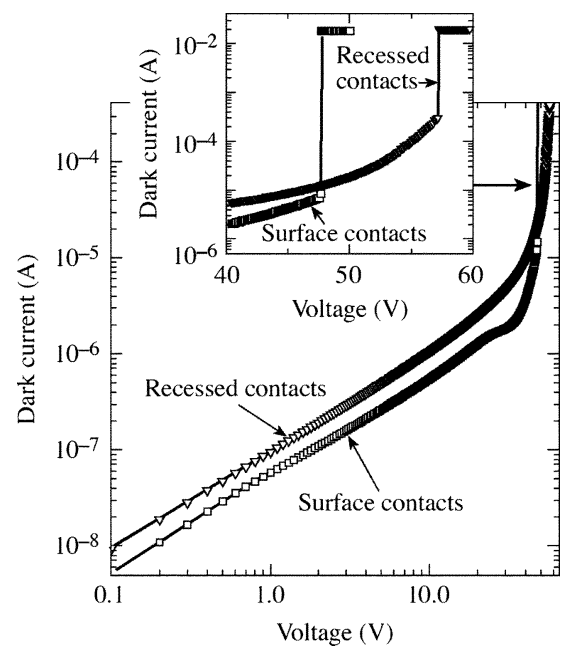

Fig. 2. I-V characteristics of MSM LT-GaAs photodetectors with the recessed and planar electrodes. The recession depth was $180 \mathrm{~nm}$.

sidewalls. After fabricating our MSM structures, their entire active surface areas were coated with $200 \mathrm{~nm}$ of $\mathrm{SiO}_{2}$ and $50-\Omega$, Ti-Au coplanar strip (CPS) transmission lines with a thickness of $50 \mathrm{~nm}$ of $\mathrm{Ti}$ and $600 \mathrm{~nm}$ of Au were fabricated for electrical measurements (the MSM structure was placed between the strips at one end of the line). For the sake of comparison, MSMs with the identical geometry, but with the planar $(d=0)$ electrodes, were fabricated on the same LT-GaAs material.

We have performed both static and high-speed characterization of our recessed-electrode devices and compared them directly with the same measurements performed on the reference planar MSMs. The time-resolved photoresponse studies were performed using 100 -fs-wide 810 -nm-wavelength $82-\mathrm{MHz}$ repetition rate optical pulses from a commercial Ti : sapphire laser. The photoresponse waveforms were recorded with the help of our electrooptic sampling system, featuring $\sim 200$-fs temporal resolution [8]. Electrical transients were sampled at a spot on the CPS line located approximately $50 \mu \mathrm{m}$ away from our devices, using a movable total-internal-reflection $\mathrm{LiTaO}_{3}$ probe [8].

Fig. 2 compares the current-voltage $(I-V)$ characteristics of the photodetectors with the recessed $(d=180 \mathrm{~nm})$ and planar electrodes, both measured in the dark. We note that, in accordance with our previous investigations [9], our MSM structures with $\mathrm{Ti}-\mathrm{Au}$ electrodes exhibit an ohmic behavior for up to $\sim 40 \mathrm{~V}$ and a quadratic $I-V$ dependence at higher biases. The recessed-electrode structure exhibits a somewhat higher dark current, as compared to the regular structure, because of its extended semiconductor material volume between the electrodes. Apparently, the increased overlapping of the metal electrodes and the LT-GaAs material, together with some trap-assisted carrier generation at the sidewalls of the grooves, leads to an increase of the dark current in our recessed MSM photodetectors. However, as shown in the inset in Fig. 2, the breakdown voltage of the recessed MSM is more than $20 \%$ higher than that measured for the planar MSM. This improvement is expected and is due to the extended length of the surface path between the electrodes.

Fig. 3 shows the responsivity dependence as a function of the bias voltage for our MSM structures with $d$ varying from 0 to $300 \mathrm{~nm}$. The measurements were performed under continuous-wave (CW) 830-nm wavelength illumination. As typ-

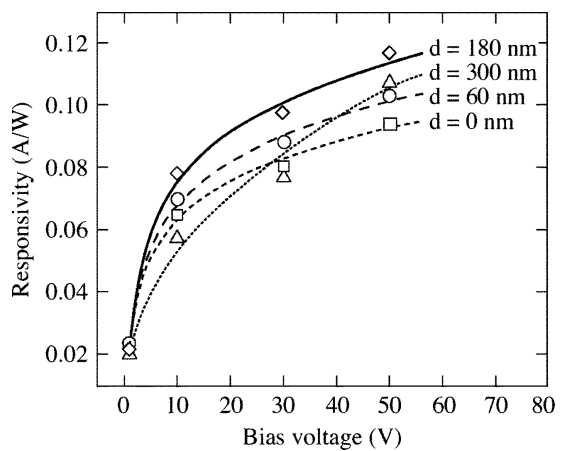

Fig. 3. Current responsivity versus the bias voltage for our MSM LT-GaAs photodetectors exhibiting several different values of the recession depth $d$.

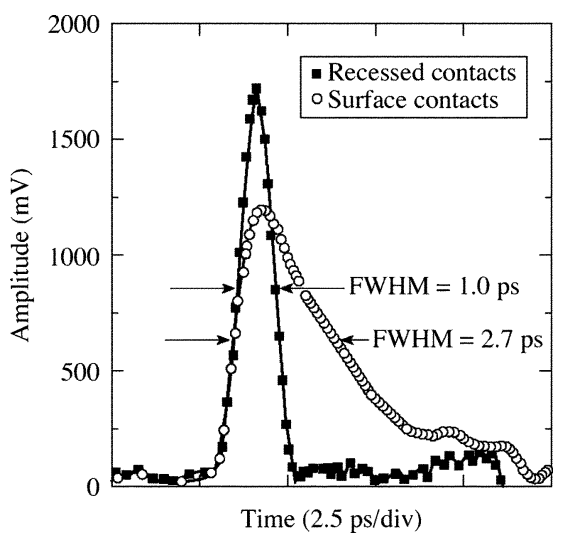

Fig. 4. Time-resolved photoresponse waveforms measured at 9-V bias for MSM photodetectors with and without recessed electrodes. The average laser power incident on the MSM was $10 \mathrm{~mW}$.

ical for MSM photodetectors, the responsivity increases with the bias increase. We note, however, that our recessed-electrode devices for large voltages exhibit an up-to-30\% increase in responsivity, as compared to the device with nonrecessed $(d=0)$ electrodes. The maximal photocurrent that was obtained for the MSM structure with the electrodes recessed $d=180 \mathrm{~nm}$ below the surface (open diamonds and solid line in Fig. 3), in which the electric field is expected to be approximately symmetric with respect to the plane of metallic electrodes. It is apparent that the recessed electrodes can better collect photogenerated carriers, leading to the substantially increased responsivity. In simple physical terms, in the recessed-electrode devices, the incident photons are absorbed in a larger LT-GaAs volume, where the relatively uniform, high electric field assures the most efficient carrier collection. At the same time, the concentration of carriers in photogenerated regions far below the electrode surface is significantly reduced. We need to stress, however, that when the recessed depth becomes too large $(d=300 \mathrm{~nm}$; open triangles and dotted line in Fig. 3), the total efficiency of collecting photocarriers is decreased due to the reduced electric field near the semiconductor top surface. In such a case, the electrodes are simply buried too deep.

The time-resolved photoresponse waveforms measured for both the recessed $(d=180 \mathrm{~nm})$ and planar MSMs, biased at $9 \mathrm{~V}$, are shown in Fig. 4 . We note that the peak amplitude of the recessed-electrode device is almost $2 \mathrm{~V}$ and is $>40 \%$ larger than the photoresponse amplitude in the case of the nonrecessed device, closely following the results of the $\mathrm{CW}$ responsivity measurements, presented in Fig. 3. Simultaneously, 
the recessed-electrode MSM exhibits almost a three-times narrower full-width at half-maximum (FWHM) and a significantly shorter fall time without a slow post-pulse tail. The latter is the most important practical advantage of the recessed geometry as the transient fall time is a figure-of-merit-type parameter, commonly called the photodetector response time $\tau$. The $\tau$ values for the transients presented in Fig. 4 fitted using an exponential decay of their falling edges are equal to 3.2 and 0.9 ps for the planar and 180-nm-deep electrode structures, respectively. Both the FWHM and $\tau$ were independent of the bias voltage and the factor-of-three decrease between the planar and recessed MSMs was observed in the whole range of biases. The excellent time-domain performance of our recessed-electrode detectors, both in terms of the increased photoresponse transient amplitude (efficiency) and speed (decreased response time), as well as the lack of a "slow tail" is, again, the result of the presence of an extended semiconductor volume between the electrodes, combined with the fast and efficient collection of photocarriers in the recessed-electrode MSM geometry.

Since the carrier lifetime in LT-GaAs is $\sim 150 \mathrm{fs}$ [8], much shorter than the response times observed in our experiments (Fig. 4) and the latter values were not voltage-bias dependent, we can conclude that at least for the planar MSM, the photoresponse should be limited by the structure's capacitance. The capacitance $C$ of planar-electrode MSM structures has been studied before [10], [11] and in our particular case, using the method presented in [10], we calculated it to be approximately $52 \mathrm{fF}$. The capacitance of the recessed-electrode MSM structure is difficult to properly assess because of its complicated surface topology. One could predict that it should be higher than that for the planar case, since the semiconductor volume between the electrodes is larger; however, the electric field distributions are very different in both cases and the simple parallel-plate capacitor analogy is likely not to be correct. Thus, we measured capacitances of both the recessed and planar MSM structures, using a network analyzer. The results were obtained for a 20-GHz test frequency (the highest available to us) and we established that for the planar MSM $C=69 \pm 7 \mathrm{fF}$, while the 180-nm-recessed electrode MSM exhibited $C=19 \pm 4 \mathrm{fF}$. Thus, the recessed-electrode structure exhibits the capacitance almost four times lower than its planar counterpart, and, for LT-GaAs, we do not expect any significant changes (increase) of $C$ at the subterahertz frequencies.

The obtained values of $C$ are in very good agreement not only with the numerical value for the planar MSM obtained earlier, but, more importantly, with the experimental data shown in Fig. 4. The latter confirms that the photoresponse speed of our devices is, indeed, limited by the $R C$ time constant, where $R$ is the characteristic impedance of the CPS line. For $R$, we took the nominal, $50-\Omega$ value, as the measurements using network analyzer returned $56 \pm 12 \Omega$. Thus, the resulting $R C$ value for the planar structure is in the 3.1-3.8-ps range and for the recessed-electrode device the $R C$ varies between 0.75 and $1.2 \mathrm{ps}$. In both cases, the above $R C$ time constants coincide with the 3.2 and $0.9 \mathrm{ps} \tau$ values extracted from the transients presented in Fig. 4.

In conclusion, we have fabricated LT-GaAs photodetectors with recessed MSM electrodes. In such a design, the semiconducting material is both above and below the plane of electrodes, leading to high and uniform electric field distribution and efficient and fast collection of optically generated carriers. The above geometry resulted in a more efficient (higher responsivity) and lower capacitance (faster) MSM photodetector. We observed the significant performance improvement of our recessed-electrode MSMs in their time-resolved photoresponse, as compared to standard planar-electrode MSM structures, tested under exactly the same conditions. Our recessed-electrode devices exhibited 1-ps-wide photoresponse transients (the decrease by almost the factor of three) with $40 \%$ higher amplitudes. The falling edge of the signal was approximately four times shorter and without the slow post-pulse tail or shoulder always observed in planar MSM diodes. The decrease of the photoresponse time of our devices we associate with their significantly reduced capacitance, while the increase in the photoresponse amplitude and the lack of the slow tail are due to more-efficient/faster collection of carriers because of the extended high electric field region. The presented recessed-electrode structures represent a step forward in the MSM-type photodetector design, as they are very suitable for ultrafast and high-sensitivity applications.

\section{ACKNOWLEDGMENT}

The authors would like to thank L. Zhang and H. Wu for their assistance in the network analyzer measurements.

\section{REFERENCES}

[1] D. C. Look, "Molecular-beam epitaxial GaAs grown at low-temperatures," Thin Solid Films, vol. 231, pp. 61-73, Aug. 1993.

[2] E. R. Brown, F. W. Smith, and K. A. McIntosh, "Coherent millimeterwave generation by heterodyne conversion in low-temperature-grown GaAs photoconductors," J. Appl. Phys., vol. 73, pp. 1480-1484, Feb. 1993.

[3] M. Y. Liu, S. Y. Chou, T. Y. Hsiang, S. Alexandrou, and R. Sobolewski, "Nanoscale metal-semiconductor-metal photodetectors with subpicosecond response-time fabricated using electron-beam lithography," $J$. Vac. Sci. Technol. B, vol. 10, pp. 2932-2935, Nov./Dec. 1992.

[4] P. Kordoš, A. Förster, M. Marso, and F. Ruders, " $550 \mathrm{GHz}$ bandwidth photodetector on low-temperature grown molecular-beam epitaxial GaAs," Electron. Lett., vol. 34, pp. 119-120, Jan. 1998.

[5] L. H. Laih, T. C. Chang, Y. A. Chen, W. C. Tsay, and J. W. Hong, "Characteristics of MSM photodetectors with trench electrodes on P-type Si wafer," IEEE Trans. Electron Devices, vol. 45, no. 9, pp. 2018-2023, Sep. 1998.

[6] M. Mikulics, "Preparation and Optimization of Low-Temperature-Grown GaAs Photomixers," Ph.D. Thesis, Research Center Jülich and RWTH Aachen, 2004.

[7] J. Waclavek, G. Krausko, and J. Škriniarová, "Optical in situ monitoring of wet chemical etching," Surf. Interface Anal., vol. 26, pp. 56-61, Jan. 1998.

[8] X. Zheng, Y. Xu, R. Sobolewski, R. Adam, M. Mikulics, M. Siegel, and P. Kordoš, "Femtosecond response of a free-standing LT-GaAs photoconductive switch," Appl. Opt., vol. 42, pp. 1726-1731, Mar. 2003.

[9] P. Kordoš, M. Marso, and M. Luysberg, "Conduction in nonstoichiometric molecular-beam epitaxial GaAs grown above the critical thickness," Appl. Phys. Lett., vol. 72, pp. 1851-1853, Apr. 1998.

[10] Y. C. Lim and R. A. Moore, "Properties of alternately charged coplanar parallel strips by conformal mappings," IEEE Trans. Electron Devices, vol. ED-15, no. 3, pp. 173-179, Mar. 1968.

[11] C. Veyres and V. F. Hanna, "Extension of the application of conformal mapping techniques to coplanar lines with finite dimensions," Int. J. Electron., vol. 48, pp. 47-56, 1980.

[12] R.-H. Yuang, Y.-J. Chien, J.-L. Shieh, and J.-I. Chyi, "High-speed GaAs metal-semiconductor-metal photodetectors with recessed metal electrodes," Appl. Phys. Lett., vol. 69, pp. 245-247, Jul. 1996.

[13] R.-H. Yuang, J.-L.Shieh, J.-I. Chyi, and J.-S. Chen, "Overall performance improvement in GaAs MSM photodetectors by using recessed-cathode structure," IEEE Photon. Technol. Lett., vol. 9, no. 2, pp. 226-228, Feb. 1997. 